\title{
Factores Moduladores de las Respuestas de los Infantes al Paradigma Still-Face: Una Revisión Sistemática
}

\section{Modulating Factors of Infants' Responses to the Still-Face Paradigm: A Systematic Review}

\author{
Lucas Gustavo Gago Galvagno ${ }^{1,2,3}$, María Carolina De Grandis ${ }^{1,2}$, \\ Ángel Manuel Elgier ${ }^{1,2,3}$ y Alba Elisabeth Mustaca ${ }^{1}$ \\ ${ }^{1}$ Facultad de Psicología y Relaciones Humanas, Universidad Abierta Interamericana \\ ${ }^{2}$ Instituto de Investigaciones, Facultad de Psicología, Universidad de Buenos Aires \\ ${ }^{3}$ Laboratorio de Cognición y Políticas Públicas, Consejo Nacional de Investigaciones Científicas y Técnicas
}

\begin{abstract}
El paradigma still-face (PSF) es un método de evaluación en 3 fases de la conducta de los bebés: luego de un período de juego libre entre madre-infante, súbitamente la madre mantiene una cara neutra, dejando de responder al niño, para finalmente reanudar el juego. Es utilizado para evaluar la regulación emocional y el comportamiento social del niño. Se realizó una revisión sistemática de artículos en revistas, usando las bases de datos Psycinfo, Scopus y EBSCO, recabando estudios sobre los principales factores que modulan las respuestas al PSF. De los artículos que contenían las palabras clave utilizadas, 131 cumplieron los criterios de inclusión. Los resultados arrojaron que los factores moduladores más estudiados fueron la sensibilidad materna, la presencia de trastornos psíquicos en la madre y el temperamento del infante. En todos los estudios se halló en los infantes un patrón de respuesta similar. Se concluye que existe robustez del paradigma. Se señala que son necesarios más estudios sobre la influencia del nivel socioeconómico, en edades más avanzadas, en países de África, Asia y Latinoamérica, y estudios longitudinales que evalúen los cambios de respuesta durante el desarrollo. Los resultados hallados son compatibles como para considerar al PSF como un modelo de frustración social en infantes.
\end{abstract}

Palabras clave: paradigma still-face, regulación emocional, frustración social, infancia

The still-face paradigm (SFP) is a 3-phase method for evaluating the behavior of babies in which, after a period of free play between mother-infant, the mother suddenly adopts a neutral expression and stops responding, but continues playing after a while. This method is used to assess the child's emotional regulation and social behavior. A systematic review of journal articles was conducted using the Psycinfo, Scopus, and EBSCO databases, which yielded studies on the main factors that modulate responses to the SFP. Of all the articles that contained the keywords used, 131 met the inclusion criteria. Results showed that the most studied modulating factors were maternal sensitivity, the presence of psychic disorders in the mother, and the temperament of the infant. A similar response pattern was found in infants in all studies, indicating that the paradigm is robust. It is pointed out that more studies need to be conducted on the influence of socioeconomic status, in older children, in countries of Africa, Asia, and Latin America, as well as longitudinal studies that evaluate the changes in response during child development. The results found are sufficiently compatible to consider the SFP as a model of social frustration in infants.

Keywords: still-face paradigm, emotion regulation, social frustration, infancy

\section{Paradigma Still-Face}

El paradigma face-to-face still-face (en adelante, PSF) ha sido utilizado durante más de 25 años para evaluar los efectos emocionales, comportamentales y fisiológicos que ocurren cuando la madre (u otros) ignora al infante en un contexto en el cual estaba estimulado socialmente, para luego retomar su interacción (Tronick et al., 1978).

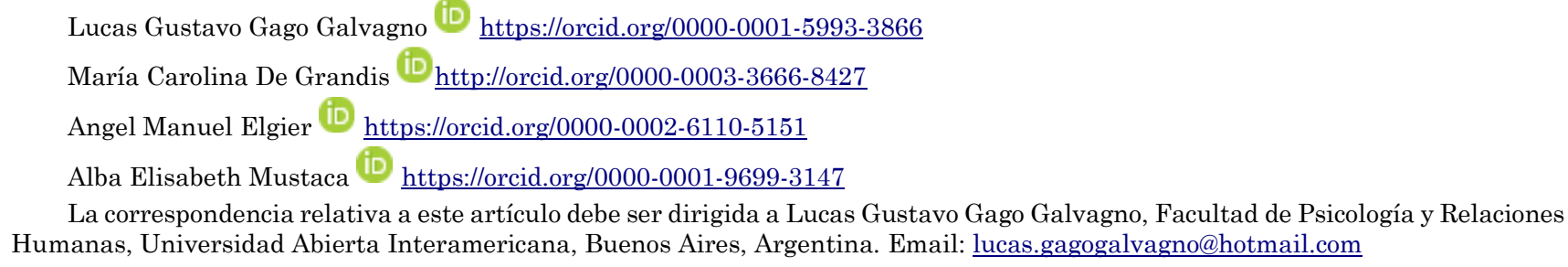


Específicamente, el PSF se lleva a cabo en tres fases consecutivas con la madre (y en menor medida con el padre) y el niño colocados frente a frente. En la primera etapa de face-to-face (fase I), hay una interacción activa entre la madre y el niño en la cual hay contacto visual y táctil y sonrisas; luego le sigue el período stillface (fase II), en que la madre súbitamente deja de interactuar con el infante (se queda con una expresión neutra en su rostro). Por último, comienza el período de reunión (fase III), en el cual la madre retoma la interacción con su hijo al igual que en la fase I. En infantes de 9 meses en adelante suele agregarse al procedimiento un conjunto de juguetes y las pruebas se llevan a cabo colocando una alfombra en el piso donde el niño y su madre interactúan (Weinberg et al., 2008). La duración de cada fase oscila entre 90 y 120 segundos y estas secuencias se filman para su posterior análisis. En la mayoría de las investigaciones se utiliza un diseño intrasujeto ABA (fase I, II, III), en el cual se evalúa la conducta de los niños y eventualmente de la madre (y en algunos casos respuestas fisiológicas) en función de las tres fases. En el trabajo de DiCorcia et al. (2016) se incluyó un grupo control AAA en el cual los niños y las madres interactuaban constantemente (solo fase I) para comparar los resultados con el grupo experimental ABA.

Durante la fase I los infantes tienen respuestas emocionales positivas (e.g., sonríen, miran a la madre; Tronick et al., 1978). En la fase II, ante la pasividad y neutralidad de la madre, sus respuestas cambian a una ausencia o disminución del afecto positivo (e.g., sonrisas), un incremento del afecto negativo (e.g., llanto y vocalizaciones con tono negativo), no mirar a la madre, darse vuelta, entre otras (Tronick et al., 1978). Otro tipo de comportamientos que se observaron fue un incremento en la actividad motora y en la manipulación de objetos (Mesman et al. 2009). A nivel fisiológico, mostraron un aumento de su ritmo cardíaco (Field, 1984) y de los niveles de cortisol en saliva (Grant et al., 2009; Ramsay \& Lewis, 2003) y un descenso de su tono vagal, a diferencia de las madres, en que el tono vagal aumenta respecto de la fase anterior (Moore et al., 2009). Durante la fase III las conductas de los niños se van recuperando parcial y paulatinamente, mostrando respuestas semejantes a las de la fase I, aunque nunca llegan a alcanzar sus valores (Adamson \& Frick, 2003; Tronick et al., 1978). En el diseño de dos grupos (experimental y control) de DiCorcia et al. (2016) se halló que durante la fase I no había diferencias entre los grupos en la duración y frecuencia de la mirada del niño hacia la madre, pero sí la hubo durante la fase II, observándose una caída abrupta y significativa que se fue recuperando de modo gradual en la fase III, aunque no llegó a los mismos niveles del grupo control. A esta última fase se la llama también Carry-over effect porque se considera que el infante no se recupera totalmente, porque hay una continuación del estrés causado en la fase II (Tronick et al., 1978).

El PSF fue aplicado mayormente durante el primer año de vida, porque en ese rango etario comienzan a desarrollarse conductas de autorregulación y permite realizar intervenciones que logren optimizarlas para potenciar su desarrollo posterior (Yoo \& Reeb-Sutherland, 2013). Hubo unas pocas evaluaciones desde los primeros días de vida. La investigación longitudinal pionera de Tronick et al. (1978) ha mostrado estabilidad en las respuestas de los niños entre 1 a 4 meses en las fases II y III. Los resultados fueron diferentes al evaluar bebés recién nacidos. Bertin y Striano (2006) hallaron la respuesta típica del PSF a los 1, 5 y 3 meses, pero no así en los de aproximadamente 4 días. Además, Bigelow y Power (2012) encontraron que los niños de una semana mostraron una continuación del afecto negativo (carry-over effect) durante la fase III, a partir de la medición de la aversión a la mirada hacia la madre, reduciéndose con respecto a la fase I.

Este método se ha utilizado para distintos propósitos. En el ámbito teórico, se ha usado para evaluar el desarrollo evolutivo de las respuestas del niño, en particular de sus capacidades emocionales, autorregulatorias y comunicativas, y la influencia de factores ambientales y del temperamento. La capacidad del niño para regular sus emociones en el PSF se evalúa a través de sus respuestas durante las fases II y III. Se considera que un niño tiene una adecuada autorregulación emocional cuando en la fase II mantiene niveles bajos de afectividad negativa (cantidad de quejas, llantos y gritos), de conductas agresivas (golpear la mesa, mover el cuerpo y los brazos), altos niveles de interacciones (señalar o llamar a la madre) y conductas de autoconsuelo (llevarse el dedo a la boca, jugar solo o quedarse quieto). Durante la fase III, una vez restablecida la interacción, la regulación emocional se mide en función de la duración e intensidad de las respuestas negativas y positivas (carry-over effect). En el ámbito clínico se utilizó como test de screening, para evaluaciones generales sobre las conductas de interacción entre padres e infantes y descubrir deficiencias en la regulación emocional, y como tratamiento para promover la sensibilidad parental y de regulación emocional de los niños con desarrollo atípico, mediante la observación y análisis de sus respuestas, a través de videos compartidos en conjunto entre clínicos y padres (Giusti et al., 2018; Wagner et al., 2016).

En conclusión, el patrón de respuestas de los niños ante el PSF fue similar en la mayoría de los niños a partir del mes de nacimiento, independientemente del contexto donde fue aplicado (Adamson \& Frick, 2003) y del sexo (e.g., Busuito \& Moore, 2017; Mesman et al., 2009; Vieites \& Reeb-Sutherland, 2017). Estos 
resultados sugieren que las respuestas de los infantes al PSF presentan características comunes en todos los infantes evaluados, aunque varían en su intensidad y duración (Weinberg et al., 2008), especialmente durante la fase II y su recuperación en la fase III.

\section{Modelos Teóricos Propuestos y Factores Reguladores de los Efectos del PSF}

En cuanto a las interpretaciones de los efectos provocados por el PSF, una teoría alternativa es la propuesta por Field (1984). Considera que los padres son un importante modulador de la regulación emocional infantil. Por ende, si ellos no están habilitados para la interacción, la sincronía se pierde y las emociones del infante se desregulan. Field (1984) utiliza como ejemplo las investigaciones de este paradigma en relación con la interacción con padres que presentan diagnóstico de depresión. Los niños de madres con depresión muestran un mayor afecto positivo durante las fases II y III. Field (1984) interpretó esos resultados como que ya de por sí las interacciones cotidianas de la madre poseen un mayor afecto neutro o negativo y hay menores interacciones con los infantes, por consecuencia, pueden acostumbrarse al comportamiento neutro de sus madres y reaccionan de la manera que consideran apropiada con ella (Aktar et al., 2017; Apter et al., 2017; Crandell et al., 2003; Graham et al., 2018). En estos casos los infantes se enfrentan a una falta constante de un regulador externo, que en este caso son sus cuidadores. Los padres serían, según Field (1984), importantes reguladores de la conducta de sus hijos, y si ellos no interactúan adecuadamente con el niño, la sincronía se pierde. Lo mismo se observa en las investigaciones que evalúan los niveles de sensibilidad materna (SM). Cuando la misma es baja, aparecen mayores niveles de desregulación emocional durante la fase III, expresados en mayores niveles de afecto negativo y una disminución del afecto positivo (Conradt \& Ablow, 2010; Kogan \& Carter, 1996).

Werner y Gross (2010) interpretan las respuestas de los infantes en el PSF como estrategias de regulación emocional; se definen como un proceso que sirve para incrementar, disminuir o mantener una o más facetas de las emociones y están influidas por numerosas variables biopsicosociales. La autorregulación de los afectos negativos, como el que se activa en la fase II en los niños en el PSF, son fundamentales para el desarrollo posterior (Miller \& Marcovitch, 2015) y su estudio permite generar teorías sobre el desarrollo infantil y plantear agendas futuras de trabajo.

Trevarthen (1977) y su concepto de intersubjetividad daría cuenta de la importancia de los padres para comprender la regulación emocional en niños. La madre monitorea las reacciones de sus hijos, los imita y adapta sus niveles de estimulación a la expresividad de ellos, fomentando, de este modo, el desarrollo de las competencias emocionales del niño. Cuando las intenciones de la madre son paradójicas, como en el caso de la fase II, la regulación del niño decae y aparece la confusión y el afecto negativo (Mesman et al., 2009). A interpretaciones similares arribó Fogel (1982), estableciendo que, a medida que el niño se desarrolla, se vuelve más capaz de tolerar altos niveles de activación emocional (arousal) ante la falta de interacción. Aunque no se encontraron diferencias en las respuestas típicas a la fase II, a medida que la edad de los niños aumenta, muestran mayores capacidades para utilizar la coordinación de la mirada y la acción de las manos, lo que sugiere que las habilidades cognitivas y motoras influencian las respuestas emocionales durante esta fase. A mayor edad, el niño realiza más respuestas directivas y activas durante la fase II (Toda \& Fogel, 1993). En la misma línea, en las dos investigaciones con el PSF llevadas a cabo en niños de 2 años, si bien se encontraron los mismos tipos de respuesta, ellos utilizaron su protolenguaje para significar aquello que estaban presenciando, además de señalamientos y juguetes, posiblemente para llamar la atención de la madre (Gago Galvagno et al., 2019; Weinberg et al., 2008).

Teniendo en cuenta estas teorías, se puede hipotetizar que el PSF: (1) es una respuesta esperable y consistente en todos los niños sanos (en la fase II y III), (2) debe aparecer a edades tempranas y (3) provoca respuestas que pueden estar reguladas en su intensidad y duración por factores individuales y contextuales (en mayor parte en la fase III y muy poco en la fase II). Los resultados presentados en los párrafos anteriores apoyan las hipótesis 1 y 2 . Respecto de la hipótesis 3, se realizaron investigaciones sobre las variables que influyen el PSF; sin embargo, no se encontraron revisiones sistemáticas actualizadas sobre este tema. La última fue la realizada por Mesman et al. (2009) y el metaanálisis de Graham et al. (2018), que fue parcial, porque solamente evaluó la influencia de madres depresivas en las respuestas de los niños al PSF. Ambos trabajos apoyan la hipótesis 3, aunque faltaría actualizar los estudios posteriores al 2009 para evaluar la consistencia de los resultados y, en especial, de los factores reguladores del PSF.

El propósito de este estudio fue realizar una revisión sistemática que recabó las investigaciones realizadas desde el 2009 hasta la fecha sobre el PSF, con los objetivos de: (a) analizar los factores que modulan 
las respuestas de los infantes ante el PSF y (b) evaluar la robustez del paradigma. Para ello se analizaron los factores moduladores de este paradigma que aún poseen inconsistencias, los que precisan mayores estudios y los que no han sido evaluados, resaltando, además, la robustez del paradigma con el fin de confirmar si puede considerarse un modelo apropiado para evaluar los efectos de la exclusión social en infantes.

\section{Método}

\section{Criterios de Elegibilidad}

Esta revisión se basó en las guías propuestas por la metodología PRISMA para la presentación de informes de revisiones sistemáticas (Celestino \& Bucher-Maluschke, 2018). Esta metodología implica anticipar los criterios de selección de los trabajos a incluir. Se revisaron las investigaciones realizadas en la última década (2009-2019), reportadas en artículos de revistas en los idiomas de inglés, portugués y español, con el estatus de artículo publicado.

\section{Fuentes de Información}

Se utilizaron las bases de datos Psycinfo, Scopus y EBSCO, incluyendo la combinación de las siguientes palabras clave en inglés, portugués y español: still-face paradigm, still-face, carry-over effect, positive affect, negative affect, face-to-face still-face, face-to-face, mother-infant interaction, infant y toddlers. Para asegurar la saturación de la literatura, se aplicaron estrategias de búsqueda hacia adelante (buscar artículos que citen el estudio incluido) y hacia atrás (revisar las listas de referencia de los estudios incluidos). Se revisaron, además, las listas de referencias de las revisiones sistemáticas y metaanálisis sobre el tema.

El esquema de la búsqueda realizada fue el siguiente: "Still-Face" OR "Still-Face Paradigm" OR "Faceto-Face Still-Face") AND ("Carry-over Effect" OR "Positive Affect" OR "Negative Affect" OR "Self-comforting") AND ("Mother-infant Interaction" OR "Infant" OR "Toddlers").

\section{Selección de Estudios}

Se llevó a cabo la lectura del título, resumen y palabras clave de los artículos. Las características que debían cumplir los artículos fueron: (a) infantes con desarrollo típico, (b) investigaciones con poder asociativo o explicativo (no descriptivas o exploratorias), (c) con variables externas o internas de los infantes o familiares que evalúen su relación con los comportamientos del paradigma still-face, (d) que evalúen principalmente los comportamientos del infante de afectividad positiva y negativa, la aversión a la mirada y el autoconsuelo. No hubo criterios de exclusión en cuanto a los adultos participantes de las díadas de interacción. Se descartaron las investigaciones que analizaban únicamente la conducta materna o únicamente variables psicofisiológicas como variable dependiente.

En el caso de que estos criterios de inclusión no fueran cumplidos o que la mera lectura del título, resumen y palabras clave no fuesen suficientes, se accedía a leer el artículo completo, analizando finalmente si cumplía con los requisitos de la revisión.

En los casos en que no se pudo acceder al artículo directamente, se contactó a los autores a través de las redes sociales especializadas (ResearchGate, Academia.com, entre otras) o por correo electrónico. En la Tabla 1 se resumen los resultados principales de cada uno de los estudios de fuente primaria. La tabla se organizó según la principal variable independiente teórica y el año de publicación. Los primeros 15 artículos $(48,4 \%)$ tienen como principal variable reguladora del comportamiento la SM, los 9 siguientes (29\%), la presencia de trastornos o síntomas de trastornos en las madres, los 3 posteriores $(9,7 \%)$, variables sociodemográficas ligadas a las familias (e.g., nivel educativo, nivel socioeconómico [NSE]). Finalmente, los últimos $4(12,9 \%)$ tienen como principal variable independiente el temperamento infantil.

De los 31 estudios revisados, el 48,4\% pertenecían a Norteamérica, el 35,5\%, a Europa, el 9,7\%, a Latinoamérica, el 3,2\%, a Oceanía y el 3,2\%, a Asia. En los cinco primeros años de la década estudiada (20092014), se encontraron 17 trabajos. En los últimos cinco años (2015 a 2019) se publicaron 14 artículos, el 45,2\% del total de todos los recogidos e incluidos en esta investigación. Esto indica que el interés por el tema se mantiene relativamente estable en los 10 años que abarca esta revisión. 
Tabla 1

Resumen de Investigaciones Empíricas que Evaluaron el Efecto de Distintos Factores Moduladores sobre el PSF

\begin{tabular}{|c|c|c|c|}
\hline \multirow{2}{*}{ Estudio } & \multirow{2}{*}{ País } & \multirow{2}{*}{ Muestra } & Resultados principales \\
\hline & & & Sensibilidad materna \\
\hline Moore et al. (2009) & $\begin{array}{l}\text { Estados } \\
\text { Unidos }\end{array}$ & $\begin{array}{l}\text { Díadas madre-infante de } 6 \text { meses } \\
\qquad(n=152) \\
\text { varones }=77 / \text { mujeres }=75\end{array}$ & $\begin{array}{l}\text { Niveles altos de sensibilidad materna durante sesiones de juego libre, reducían el tono vagal de la madre y el } \\
\text { niño, medidos como arritmia sinusal respiratoria (ralentización fisiológica de la frecuencia cardíaca) durante la } \\
\text { fase III. No se encontraron diferencias en el afecto positivo y negativo según la sensibilidad materna y el nivel } \\
\text { socioeconómico de los infantes. Se halló la respuesta típica al PSF. }\end{array}$ \\
\hline $\begin{array}{l}\text { Conradt \& Ablow } \\
\quad(2010)\end{array}$ & $\begin{array}{l}\text { Estados } \\
\text { Unidos }\end{array}$ & $\begin{array}{l}\text { Díadas madre-infante de } 5 \text { meses } \\
\text { de bajo nivel socioeconómico } \\
\qquad(n=95) \\
\text { varones }=42 / \text { mujeres }=53\end{array}$ & $\begin{array}{l}\text { La sensibilidad materna predijo la reactividad y regulación biocomportamental de los infantes durante la fase II. } \\
\text { Con altos niveles de sensibilidad materna, el ritmo cardíaco de los infantes disminuía en las tres fases. No se } \\
\text { encontraron diferencias con respecto a la arritmia sinusal respiratoria. En cuanto al comportamiento, esos } \\
\text { mismos bebés mostraron mayores niveles de interacción atencional y menos conductas de afectividad negativa en } \\
\text { la fase III, respecto de madres con mayor sensibilidad. Se halló la respuesta típica al PSF. }\end{array}$ \\
\hline Grant et al. (2010) & Australia & $\begin{array}{l}\text { Díadas madre-infante de } 7 \text { meses } \\
\qquad(n=84) \\
\text { No se especifica sexo }\end{array}$ & $\begin{array}{l}\text { La sensibilidad materna se asoció inversamente únicamente con la afectividad negativa y directamente con la } \\
\text { afectividad positiva durante la fase III. Se halló la respuesta típica al PSF. }\end{array}$ \\
\hline $\begin{array}{l}\text { Bigelow \& Power } \\
\quad(2012)\end{array}$ & Canadá & $\begin{array}{l}\text { Díadas madre-infante desde } 1 \\
\quad \begin{array}{l}\text { semana a } 3 \text { meses } \\
\quad(n=60)\end{array} \\
\text { varones }=28 / \text { mujeres }=32\end{array}$ & $\begin{array}{l}\text { Niveles altos de respuesta materna en fase I se asociaron a mayores interacciones entre la díada madre e hijo y } \\
\text { mayores niveles de respuesta y expectativas para la interacción social de los infantes durante la fase III a los } \\
2 \text { y } 3 \text { meses. Sin embargo, estos niveles altos no contribuían a la afectividad positiva a lo largo de las fases en } \\
\text { ninguna etapa del desarrollo. Se halló la respuesta típica al PSF. }\end{array}$ \\
\hline Lowe et al. (2012) & $\begin{array}{l}\text { Estados } \\
\text { Unidos }\end{array}$ & $\begin{array}{l}\text { Díadas madre-infante de } 3 \frac{1 / 2}{\text { a }} 4^{1 / 2} \\
\text { meses } \\
\quad(n=70) \text { y } 9 \text { meses }(n=25) \\
\text { No se especifica sexo }\end{array}$ & $\begin{array}{l}\text { Los hijos de las madres que mostraron ser más responsivas (mayor sensibilidad a las interacciones) mostraron } \\
\text { mayor capacidad de regulación emocional evidenciada por mayor afecto positivo durante las fases I y III. En } \\
\text { contraste, los infantes de madres con menores niveles de responsividad ante las interacciones mostraron menor } \\
\text { capacidad de regulación emocional. Poseían menores niveles de afecto positivo en las fases I y III. Se halló la } \\
\text { respuesta típica al PSF. }\end{array}$ \\
\hline $\begin{array}{l}\text { Gunning et al. } \\
\quad(2013)\end{array}$ & $\begin{array}{l}\text { Reino } \\
\text { Unido }\end{array}$ & $\begin{array}{l}\text { Díadas madre-infante de } 3 \text { meses } \\
\qquad \begin{array}{l}(n=121) \\
58 \text { madres de alto nivel de riesgo y } \\
63 \text { de bajo } \\
\text { varones }=57 / \text { mujeres }=64\end{array}\end{array}$ & $\begin{array}{l}\text { Mayores niveles de sensibilidad materna se asociaron a menores niveles de afecto negativo durante las fases I y } \\
\text { II, pero no en la III. La sensibilidad materna no impactó en el ritmo cardíaco en ninguna de las fases. Solo se } \\
\text { hallaron diferencias por grupo en la cantidad de conductas de afecto negativo en la fase II y en los niveles de } \\
\text { sensibilidad materna: las madres en situación de riesgo presentaron menores niveles de sensibilidad materna. } \\
\text { No se encontraron diferencias en el resto de las fases. Se halló la respuesta típica al PSF. }\end{array}$ \\
\hline
\end{tabular}




\section{Tabla 1 (Continuación)}

Resumen de Investigaciones Empíricas que Evaluaron el Efecto de Distintos Factores Moduladores sobre el PSF

\begin{tabular}{|c|c|c|c|}
\hline \multirow{2}{*}{ Estudio } & \multirow{2}{*}{ País } & \multirow{2}{*}{ Muestra } & Resultados principales \\
\hline & & & Sensibilidad materna \\
\hline $\begin{array}{l}\text { Bigelow \& Power } \\
\quad(2014)\end{array}$ & Canadá & $\begin{array}{l}\text { Díadas madre-infante desde } 1 \\
\quad \text { semana a } 3 \text { meses } \\
\qquad(n=60) \\
\text { varones }=28 / \text { mujeres }=32\end{array}$ & $\begin{array}{l}\text { A partir de los } 2 \text { meses los infantes sonreían y generaban vocalizaciones sin estrés cuando miraban a sus madres } \\
\text { durante la fase II. Estas conductas se correlacionaron directamente con los niveles de respuesta de la madre, } \\
\text { específicamente con respuestas vocales contingentes y con comportamientos sociales positivos en la fase I de } \\
\text { interacción. Las respuestas mediante vocalizaciones por parte de la madre fueron el predictor más importante de } \\
\text { estos comportamientos del infante a los } 2 \text { y } 3 \text { meses en la fase II. Se halló la respuesta típica al PSF. }\end{array}$ \\
\hline $\begin{array}{l}\text { Braungart-Rieker } \\
\text { et al. (2014) }\end{array}$ & Italia & $\begin{array}{l}\text { Triadas madre-padre-infante de } 3, \\
\quad 5 \text { y } 7 \text { meses } \\
(n=135) \\
\text { varones }=64 / \text { mujeres }=71\end{array}$ & $\begin{array}{l}\text { Los infantes cuyas madres y padres demostraron mayores niveles de sensibilidad, mostraron más cantidad de } \\
\text { comportamientos ligados al afecto positivo, orientación de mirada hacia los padres y autoconsuelo en las } \\
\text { fases II y III, siendo mayor la asociación a los } 7 \text { meses. Con respecto al afecto negativo, la correlación fue inversa } \\
\text { y se encontró en todas las edades evaluadas. Se halló la respuesta típica al PSF. }\end{array}$ \\
\hline $\begin{array}{l}\text { MacLean et al. } \\
\quad(2014)\end{array}$ & $\begin{array}{l}\text { Estados } \\
\text { Unidos }\end{array}$ & $\begin{array}{l}\text { Díadas madre-infante de } 4 \text { meses } \\
\qquad(n=84) \\
\text { varones }=48 / \text { mujeres }=36\end{array}$ & $\begin{array}{l}\text { El afecto positivo de los infantes durante las fases I y III estuvo asociado con interacciones de mirada entre } \\
\text { la madre e infante (sincronía) un segundo después de que las mismas ocurrieran. Se halló la respuesta típica } \\
\text { al PSF. }\end{array}$ \\
\hline $\begin{array}{l}\text { Mastergeorge et al. } \\
\quad(2014)\end{array}$ & $\begin{array}{l}\text { Estados } \\
\text { Unidos }\end{array}$ & $\begin{array}{l}\text { Díadas madre-infante de } 1 \text { mes de } \\
\text { bajo nivel socioeconómico } \\
\qquad(n=31) \\
\text { varones }=13 / \text { mujeres }=18\end{array}$ & $\begin{array}{l}\text { Mayores niveles de sensibilidad materna predijeron menores niveles de afecto negativo del niño en el período de } \\
\text { reunión. No se encontraron diferencias en el período still-face. Se halló la respuesta típica al PSF. }\end{array}$ \\
\hline $\begin{array}{l}\text { Coppola et al. } \\
\quad(2016)\end{array}$ & Italia & $\begin{array}{l}\text { Díadas madre-infante de } 6 \text { meses } \\
\qquad \begin{array}{l}(n=71) \\
\text { varones }=35 / \text { mujeres }=36\end{array}\end{array}$ & $\begin{array}{l}\text { Las madres con mayores niveles de sensibilidad materna demostraron mayor cantidad de interacciones de juego } \\
\text { en las tres fases y menor cantidad de conductas que fueron tipificadas como neutrales (falta de respuesta) e } \\
\text { intrusivas (intervenir el juego del niño). Se halló la respuesta típica al PSF. }\end{array}$ \\
\hline $\begin{array}{l}\text { Busuito \& Moore } \\
\quad(2017)\end{array}$ & $\begin{array}{l}\text { Estados } \\
\text { Unidos }\end{array}$ & $\begin{array}{l}\text { Díadas madre-infante de } 6 \text { meses } \\
\qquad \begin{aligned}(n=53) \\
\text { varones }=33 / \text { mujeres }=20\end{aligned}\end{array}$ & $\begin{array}{l}\text { La sincronía y el encuentro en las interacciones diádicas se asoció con la regulación del estrés (medido a través } \\
\text { del tono vagal en el niño y los comportamientos de afectividad positiva) durante la fase III. La edad de la madre, } \\
\text { estado civil y nivel socioeconómico no se asociaron a la arritmia sinusal respiratoria del infante en ninguna de las } \\
\text { fases. Se halló la respuesta típica al PSF. }\end{array}$ \\
\hline $\begin{array}{l}\text { Handal et al. } \\
\quad(2017)\end{array}$ & Ecuador & $\begin{array}{l}\text { Díadas madre-infante de } 4 \text { meses } \\
\qquad \begin{array}{l}(n=26) \\
\text { varones }=9 / \text { mujeres }=15\end{array}\end{array}$ & $\begin{array}{l}\text { Los hijos de las madres que mostraron ser más responsivas (mayor sensibilidad a las interacciones) mostraron } \\
\text { mayor capacidad de regulación emocional evidenciada por mayor afecto positivo durante las fases I y III. En } \\
\text { contraste, los infantes de madres con menores niveles de responsividad ante las interacciones mostraron menor } \\
\text { capacidad de regulación emocional: poseían menores niveles de afecto positivo en las fases I y III. Se halló la } \\
\text { respuesta típica al PSF. }\end{array}$ \\
\hline
\end{tabular}




\section{Tabla 1 (Continuación)}

Resumen de Investigaciones Empíricas que Evaluaron el Efecto de Distintos Factores Moduladores sobre el PSF

\begin{tabular}{|c|c|c|c|}
\hline \multirow{2}{*}{ Estudio } & \multirow{2}{*}{ País } & \multirow{2}{*}{ Muestra } & Resultados principales \\
\hline & & & Sensibilidad materna \\
\hline Seixas et al. (2017) & Portugal & $\begin{array}{l}\text { Díadas madre-infante evaluados a } \\
\text { los } 3 \text { y } 9 \text { meses } \\
\quad(n=67) \\
\text { No se especifica sexo }\end{array}$ & $\begin{array}{l}\text { Los comportamientos maternales de interacción se asociaron a las respuestas infantiles en la fase III, aunque } \\
\text { hubo mayor cantidad de comportamientos de afectividad negativa en los infantes de género femenino y con } \\
\text { madres con menores niveles de sensibilidad. Se halló la respuesta típica al PSF. }\end{array}$ \\
\hline $\begin{array}{l}\text { Spinelli \& Mesman } \\
\quad(2018)\end{array}$ & Italia & $\begin{array}{l}\text { Díadas madre-infante de } 3 \text { meses } \\
\qquad(n=70) \\
\text { varones }=35 / \text { mujeres }=35\end{array}$ & $\begin{array}{l}\text { No se encontraron diferencias en los niveles de afectividad negativa en infantes de } 3 \text { meses según la variable } \\
\text { sensibilidad materna en las fases II y III. Sin embargo, cuando a la sensibilidad materna se le agregaban altos } \\
\text { niveles de lenguaje directo de la madre, sí había diferencias en la expresión de la afectividad negativa durante el } \\
\text { período de reunión. Se halló la respuesta típica al PSF. } \\
\text { Presencia de trastornos en las madres }\end{array}$ \\
\hline Kaitz et al. (2010) & Israel & $\begin{array}{l}\text { Díadas madre-infante de } 6 \text { meses } \\
\qquad(n=41) \\
34 \text { madres con trastorno de } \\
\text { ansiedad y } 59 \text { grupo control } \\
\text { No se especifica sexo }\end{array}$ & $\begin{array}{l}\text { No se encontraron diferencias en los comportamientos de los infantes con respecto a los comportamientos de las } \\
\text { madres con y sin trastornos de ansiedad durante la prueba still-face. Se halló la respuesta típica al PSF. }\end{array}$ \\
\hline $\begin{array}{l}\text { Musser et al. } \\
\quad(2012)\end{array}$ & $\begin{array}{l}\text { Estados } \\
\text { Unidos }\end{array}$ & $\begin{array}{l}\text { Díadas madre-infante de } 5 \text { meses } \\
(n=95) \text { con cantidad variable de } \\
\text { síntomas de depresión } \\
\text { No se especifica sexo }\end{array}$ & $\begin{array}{l}\text { Las madres con mayor cantidad de síntomas de depresión mostraron menores niveles de sensibilidad materna. } \\
\text { Sin embargo, no hubo diferencias en las formas de interacción madre-infante durante el PSF. Se halló la respuesta } \\
\text { típica al paradigma still-face. }\end{array}$ \\
\hline $\begin{array}{l}\text { Oppenheimer et al. } \\
\text { (2013) }\end{array}$ & $\begin{array}{l}\text { Estados } \\
\text { Unidos }\end{array}$ & $\begin{array}{l}\text { Díadas madre-infante de } 5 \text { meses } \\
(n=81) \text { con cantidad variable de } \\
\text { síntomas de depresión } \\
\text { varones }=35 / \text { mujeres }=46\end{array}$ & $\begin{array}{l}\text { Las madres con menor cantidad de síntomas de depresión mostraron mayor arritmia sinusal respiratoria en la } \\
\text { fase I, con un decaimiento en la fase II. Lo contrario se halló en madres con presencia de varios síntomas de } \\
\text { depresión, siendo menos responsivas a la demanda de los infantes. Los infantes demostraron menores niveles de } \\
\text { afectividad positiva en las tres fases. Se halló la respuesta típica al PSF. }\end{array}$ \\
\hline Noe et al. (2015) & Alemania & $\begin{array}{l}\text { Díadas madre-infante de } 1 \text { a } 8 \\
\qquad \begin{array}{l}\text { meses } \\
(n=70)\end{array} \\
\text { Muestra con depresión } \\
(n=38) \text { y control }(n=30) \\
\text { varones }=47 / \text { mujeres }=21\end{array}$ & $\begin{array}{l}\text { No hubo diferencias según la presencia de trastorno de depresión en las madres. Sin embargo, los infantes que } \\
\text { más tiempo coordinaron las emociones con las madres tuvieron mayores niveles de afectividad positiva en las } \\
\text { fases II y III. Se halló la respuesta típica al PSF. }\end{array}$ \\
\hline
\end{tabular}




\section{Tabla 1 (Continuación)}

Resumen de Investigaciones Empíricas que Evaluaron el Efecto de Distintos Factores Moduladores sobre el PSF

\begin{tabular}{|c|c|c|c|}
\hline \multirow{2}{*}{ Estudio } & \multirow{2}{*}{ País } & \multirow{2}{*}{ Muestra } & Resultados principales \\
\hline & & & Sensibilidad materna \\
\hline Apter et al. (2017) & Francia & $\begin{array}{l}\text { Díadas madre-infante de } 3 \text { meses } \\
\qquad(n=60) \\
19 \text { madres poseían diagnóstico de } \\
\text { TBP } \\
\text { varones }=22 / \text { mujeres }=38\end{array}$ & $\begin{array}{l}\text { Se hallaron diferencias en las interacciones de infantes con madres con y sin trastorno de personalidad bipolar } \\
\text { (TPB). Los infantes de madres con TPB expresaban menos vocalizaciones y miradas a la madre y menor cantidad } \\
\text { de comportamientos de autorregulación en las tres fases del PSF. También se notaron diferencias en el desempeño } \\
\text { de las madres diagnosticadas con TPB en el PSF. En el período inicial mostraron menos intercambios activos con } \\
\text { el infante y durante el período de reunión, menores niveles de afecto positivo y más momentos de intrusión. Se } \\
\text { halló la respuesta típica al PSF. }\end{array}$ \\
\hline Müller et al. (2016) & Alemania & $\begin{array}{l}\text { Díadas madre-infante de } 6 \text { meses } \\
\qquad(n=69) \\
28 \text { madres con trastorno de } \\
\text { ansiedad y } 41 \text { grupo control } \\
\text { No se especifica sexo }\end{array}$ & $\begin{array}{l}\text { Las madres con trastorno de ansiedad mostraron menos niveles de sensibilidad y sus hijos, más conductas de } \\
\text { autoconsuelo en la fase II. Sin embargo, las infantes de sexo femenino mostraron mayor sensibilidad a las } \\
\text { interacciones maternales durante la fase II. Se halló la respuesta típica al PSF. }\end{array}$ \\
\hline $\begin{array}{c}\text { Vieites \& Reeb- } \\
\text { Sutherland (2017) }\end{array}$ & $\begin{array}{l}\text { Estados } \\
\text { Unidos }\end{array}$ & $\begin{array}{l}\text { Díadas madre-infante de } 5 \text { y } 9 \\
\text { meses con niveles variados de } \\
\text { depresión no clínica } \\
\quad(n=90) \\
\text { No se especifica sexo }\end{array}$ & $\begin{array}{l}\text { A los } 5 \text { meses, los infantes de madres con mayores niveles de depresión (no clínica) tuvieron menor cantidad de } \\
\text { conductas de afecto negativo durante la fase II, en comparación con las díadas de madres con bajos niveles de } \\
\text { depresión. No se hallaron resultados a los } 9 \text { meses. Se halló la respuesta típica al PSF. }\end{array}$ \\
\hline Reck et al. (2018) & Alemania & 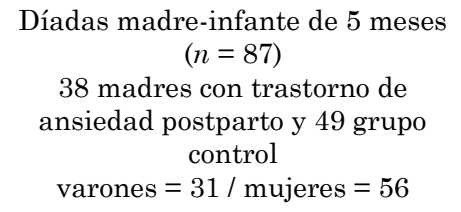 & $\begin{array}{l}\text { No hubo efectos de grupo en las fases I, II y III en afectividad positiva. Los infantes de madres con mayores } \\
\text { niveles de ansiedad postparto comenzaron con menores niveles de afecto positivo durante la fase I que los del } \\
\text { grupo control. Los bebés de madres con ansiedad mostraron mayor afectividad negativa en la fase I, aumento en } \\
\text { la fase II y mantención en la fase III. La conducta intrusiva de la madre se correlacionó directamente con el afecto } \\
\text { negativo. Se halló la respuesta típica al PSF. }\end{array}$ \\
\hline Mantis et al. (2019) & Canadá & $\begin{array}{l}\text { Díadas madre-infante de } 5 \text { meses } \\
\qquad(n=41) \\
\text { con cantidad variable de síntomas } \\
\text { de depresión } \\
\text { varones }=20 / \text { mujeres }=21\end{array}$ & $\begin{array}{l}\text { Las madres con mayor cantidad de síntomas de depresión mostraron menor cantidad de interacciones físicas } \\
\text { (tocar) en las fases I y III, en comparación con las madres sin depresión. Además, estas interacciones fueron aún } \\
\text { menores durante la fase III para el grupo de madres con depresión. No se encontraron diferencias en los } \\
\text { comportamientos infantiles. Se halló la respuesta típica al PSF. }\end{array}$ \\
\hline
\end{tabular}




\section{Tabla 1 (Conclusión)}

Resumen de Investigaciones Empíricas que Evaluaron el Efecto de Distintos Factores Moduladores sobre el PSF

\begin{tabular}{|c|c|c|c|}
\hline \multirow{2}{*}{ Estudio } & \multirow{2}{*}{ País } & \multirow{2}{*}{ Muestra } & Resultados principales \\
\hline & & & Sensibilidad materna \\
\hline & & & Nivel Socioeconómico \\
\hline Lowe et al. (2016) & Ecuador & $\begin{array}{l}\text { Díadas madre-infante de } 4 \text { meses } \\
\qquad(n=26) \\
\text { varones }=9 / \text { mujeres }=15\end{array}$ & $\begin{array}{l}\text { Los hijos de madres ecuatorianas presentaron más acercamientos afectivos, en comparación con las madres } \\
\text { hispánicas de Estados Unidos. Sin embargo, estas últimas mostraron más interacciones de juego. Estos resultados } \\
\text { se hallaron en la fase I. Se halló la respuesta típica al PSF. }\end{array}$ \\
\hline $\begin{array}{l}\text { Smaling et al. } \\
\text { (2016) }\end{array}$ & Holanda & $\begin{array}{l}\text { Díadas madre-infante de } 6 \text { meses } \\
\qquad(n=133) \\
\text { No se especifica sexo }\end{array}$ & $\begin{array}{l}\text { Mayores niveles de riesgo se asociaron inversamente con la cantidad de interacciones de la madre durante la fase } \\
\text { I. Además, la capacidad de función reflexiva de la madre (que incide sobre su sensibilidad) se asoció a mayores } \\
\text { niveles de afectividad positiva durante el período de reunión. Se halló la respuesta típica al PSF. }\end{array}$ \\
\hline \multirow[t]{2}{*}{$\begin{array}{l}\text { Gago Galvagno et } \\
\text { al. (2019) }\end{array}$} & Argentina & $\begin{array}{l}\text { Díadas madre-infante de } 18 \text { a } 24 \\
\text { meses } \\
\begin{array}{c}(n=50) \\
\text { varones }= \\
21 / \text { mujeres }=29\end{array}\end{array}$ & $\begin{array}{l}\text { El nivel educativo de los padres y el esfuerzo de control infantil se asociaron con mejores desempeños de } \\
\text { regulación emocional durante el PSF. No hubo asociaciones con el nivel socioeconómico total. Se halló la respuesta } \\
\text { típica al PSF. }\end{array}$ \\
\hline & & & Temperamento \\
\hline $\begin{array}{l}\text { Planalp et al. } \\
\text { (2013) }\end{array}$ & $\begin{array}{l}\text { Estados } \\
\text { Unidos }\end{array}$ & $\begin{array}{l}\text { Triadas padre-madre-infante de } 3 \\
\qquad \begin{array}{l}5 \text { y } 7 \text { meses } \\
(n=135)\end{array} \\
\text { varones }=64 / \text { mujeres }=71\end{array}$ & $\begin{array}{l}\text { Se encontró que la extraversión se ligaba a madres más responsivas (mayor sensibilidad materna) en la primera } \\
\text { fase del paradigma. Además, las madres eran más sensibles a las interacciones a los } 3 \text { meses; pero a los } 5 \text { y } 7 \text { meses } \\
\text { del infante mostraban los mismos niveles que el padre. No se encontraron diferencias según los estilos parentales } \\
\text { en los comportamientos del PSF. Se halló la respuesta típica al PSF. }\end{array}$ \\
\hline $\begin{array}{c}\text { Yoo \& Reeb- } \\
\text { Sutherland (2013) }\end{array}$ & $\begin{array}{l}\text { Estados } \\
\text { Unidos }\end{array}$ & $\begin{array}{l}\text { Díadas madre-infante de } 5 \text { meses } \\
\qquad(n=85) \\
\text { varones }=43 / \text { mujeres }=42\end{array}$ & $\begin{array}{l}\text { Los infantes que fueron clasificados por sus madres como altos en reactividad negativa, mostraron mayor } \\
\text { afectividad negativa durante el período de reunión, no así en la fase II. Tampoco hubo asociaciones entre } \\
\text { temperamento y regulación emocional (conductas de autoconsuelo e interacción con objetos) en ninguna de las } \\
\text { fases del paradigma. Se halló la respuesta típica al PSF. }\end{array}$ \\
\hline $\begin{array}{l}\text { Haltigan et al. } \\
\qquad(2014)\end{array}$ & $\begin{array}{l}\text { Estados } \\
\text { Unidos }\end{array}$ & $\begin{array}{l}\text { Díadas madre-infante de } 6 \text { meses } \\
\qquad(n=259) \\
\text { No se especifica sexo }\end{array}$ & $\begin{array}{l}\text { El afecto negativo de los niños tuvo una asociación inversa con las interacciones y la sensibilidad materna y una } \\
\text { asociación directa entre el afecto negativo y las retiradas en la interacción. Ambos resultados fueron hallados en } \\
\text { el episodio de reunión. Se halló la respuesta típica al PSF. }\end{array}$ \\
\hline Freund (2019) & Alemania & $\begin{array}{l}\text { Díadas madre-infante de } 8 \text { meses } \\
\qquad(n=55) \\
\text { No se especifica sexo }\end{array}$ & $\begin{array}{l}\text { A mayor nivel de afectividad negativa reportada por las madres más rápido se expresaba la afectividad negativa } \\
\text { y la irritación durante las fases II y III del PSF. Sin embargo, no midió otros estilos temperamentales. Se halló } \\
\text { la respuesta típica al PSF. }\end{array}$ \\
\hline
\end{tabular}


Todos los trabajos se publicaron en inglés, excepto uno que está en portugués (Seixas et al., 2017). Ningún estudio presentó un muestreo representativo, siendo el intencional el más recurrente. Solo el 16,1\% $(n=5)$ utilizó un diseño longitudinal. En los 20 estudios que usaron pruebas psicométricas como instrumento de recolección de información, el 80\% $(n=16)$ informó sobre la confiabilidad del instrumento por medio de alfa de Cronbach, siendo entre 0,60 y 0,70, lo cual es aceptable para este tipo de muestra (Nunnaly, 1978). Además, solo el 29\% ( $n=9)$ informó sobre el tamaño del efecto de sus resultados. En ellos, el 55,6\% $(n=5)$ obtuvo un tamaño del efecto bajo. En cuanto a la medición de las conductas, el 41,9\% $(n=13)$ de los estudios utilizó la técnica de doble ciego como control de la confiabilidad. Por último, solo un estudio trabajó con una muestra de niños mayores a 1 año (Gago Galvagno et al., 2019). En el resto de las investigaciones, la edad de los niños osciló entre 1 semana y 12 meses. Dos investigaciones incluyeron muestras de padres (BraungartRieker et al., 2014; Planalp et al., 2013); los demás fueron realizados con las madres de los infantes.

\section{Resultados}

La búsqueda inicial arrojó 187 artículos en Psycinfo, 78 en EBSCO y 185 en Scopus, de los cuales 31 cumplieron con todos los criterios mencionados. Solo fue necesario contactarse con uno de los autores para solicitarle la publicación (Freund, 2019).

\section{Factores Moduladores de las Respuestas de los Infantes al PSF}

Las investigaciones que estudian los factores que regulan el comportamiento de los niños ante el PSF, se pueden clasificar en (a) las que evalúan la influencia de factores contextuales-sociales y (b) las que se centran en las particularidades de los infantes. Entre las primeras, se estudiaron las influencias de características de la madre, en especial su SM y trastornos psicológicos y, en menor medida, factores sociodemográficos. En cuanto a las diferencias individuales de los infantes, la mayoría de las investigaciones evaluó su temperamento.

\section{Sensibilidad Materna}

La SM fue la más estudiada y con menos resultados contradictorios. La SM se define como la habilidad de la madre para tomar consciencia, interpretar adecuadamente y responder de forma apropiada y contingente a las señales y comunicaciones del infante (Ainsworth et al. 2015). En la mayoría de las publicaciones fue evaluada por la observación de los comportamientos de interacción materna en las fases del paradigma y, en menor medida, a través de escalas psicométricas autoadministradas (Bigelow \& Power, 2014; Busuito \& Moore, 2017; Kogan \& Carter, 1996; MacLean et al., 2014; Mastergeorge et al., 2014; Moore et al., 2009). Se asocia a respuestas regulatorias tanto fisiológicas como comportamentales en los infantes, que en la mayoría de los casos se expresa durante las fases II y III. Los resultados fueron consistentes: a mayor SM mayor autorregulación presentaba el infante. Además, a menor respuestas y más conductas de intromisión de las madres hacia los infantes menor autorregulación emocional se observó en los niños (e.g., Busuito \& Moore, 2017). Se puede concluir que la forma en que la madre interactúa con su hijo sería un predictor de su capacidad para autorregularse y tolerar más adecuadamente las situaciones que generan alto estrés.

\section{Trastornos Mentales de las Madres}

Nueve estudios evaluaron la presencia de problemas mentales en las madres (e.g., Aktar et al., 2017; Apter et al., 2017; Vieites \& Reeb-Sutherland, 2017). Los trastornos que se evaluaron fueron ansiedad, depresión y trastorno bipolar. No se encontraron diferencias en las respuestas de los niños de más de 5 meses durante la fase II del PSF entre madres con o sin trastornos (Mantis et al., 2019; Musser et al., 2012; Vieites \& Reeb-Sutherland, 2017). En cuanto a los menores de 5 meses, los resultados fueron contradictorios. En algunas investigaciones no se encontraron diferencias de respuestas de los niños en las tres fases del PSF según la presencia o no de trastorno de la madre (e.g., Kaitz, et al., 2010; Mantis et al., 2019; Musser et al., 2012); en otros trabajos sí se hallaron (e.g., Apter et al., 2017; Müller et al., 2016). Si bien en todos los casos las respuestas típicas de los niños en la fase II estuvieron presentes, ellas aparecieron atenuadas, ya sea presentando (en líneas generales) menores niveles de afecto positivo o mayores de negativos cuando las madres presentaban síntomas o trastornos. Sin embargo, el metaanálisis realizado por Graham et al. (2018) en niños con madres depresivas concluyó que, si bien, al igual que las otras sintomatologías, se presentó la respuesta típica de los infantes al PSF, los bebés de madres deprimidas mostraron niveles más altos de emociones positivas durante la fase II que los del grupo control. Una interpretación de los autores a esa 
respuesta fue que los infantes experimentaron interacciones similares con sus madres deprimidas y aprendieron a aumentar sus respuestas positivas de apego y contacto, porque finalmente lograban la atención de la madre.

Es de señalar que en el 55,5\% $(n=9)$ de las investigaciones en la cuales se evaluó la presencia de trastornos o síntomas en las madres, se halló que impactaron en la SM, reduciendo el número de interacciones y aumentando la cantidad de conductas intrusivas durante la interacción con el infante (e.g., Apter et al., 2017; Mantis et al., 2019; Oppenheimer et al., 2013). Esto sugiere que la SM podría ser un mediador entre la presencia de trastornos y las conductas del infante en el PSF.

\section{Factores Sociodemográficos}

El NSE general contribuyó a diferencias significativas en las interacciones entre los cuidadores e infantes, aunque los resultados no fueron consistentes. Se halló que a mayor nivel de riesgo o pobreza disminuía la cantidad de interacciones entre madre-infante durante las fases I y III (e.g., Lowe et al., 2016; Smaling et al., 2016), aunque en otros casos no se encontraron esas asociaciones (Gago Galvagno et al., 2019). La vulnerabilidad social se asoció con las conductas de las madres. Hubo menor cantidad de acercamientos físicos y verbales durante la fase I y menor responsividad en las interacciones y mayor afectividad positiva del infante durante la fase III (Lowe et al., 2016; Smaling et al., 2016).

El nivel educativo de las madres se asoció con diferencias en las interacciones durante las fases I y III: a mayor nivel educativo menor fue el nivel de afecto negativo expresado por el infante (Gago Galvagno et al., 2019) y mayores los niveles de regulación emocional general durante la fase II del paradigma (Gago Galvagno et al., 2019; Lowe et al., 2016; Smaling et al., 2016).

Todos los infantes presentaron la respuesta típica a este paradigma a pesar del NSE. Sin embargo, son escasas las investigaciones que se proponen en los últimos años analizar estas relaciones. Deberían ampliarse, ya que el fenómeno de la pobreza posee múltiples manifestaciones, dependiendo de cada tipo de cultura, por lo cual permitiría reforzar los resultados encontrados con investigaciones transculturales.

\section{Temperamento}

En lo relativo a las diferencias individuales, las respuestas al PSF aparecen moduladas principalmente por el temperamento infantil. Los menores niveles de afecto negativo y mayores de esfuerzo de control y de extraversión de los infantes se asociaron a mayores niveles de afectividad positiva e interacciones en las fases II y III (Gago Galvagno et al., 2019; Yoo \& Reeb-Sutherland, 2013). Además, el temperamento del infante se asoció con respuestas de interacción de la madre (Planalp et al., 2013).

Sin embargo, no todas las investigaciones hallaron asociaciones con el temperamento (Gago Galvagno et al., 2019; Yoo \& Reeb-Sutherland, 2013) y todas utilizaron medidas indirectas del temperamento (escalas psicométricas de autoinforme parental) y no medidas conductuales directas. A pesar de esto, todos los infantes presentaron la respuesta típica a este paradigma, independientemente del estilo temperamental.

\section{Robustez del Paradigma}

Todas las investigaciones presentaron un patrón de respuesta similar ante el PSF en todos los infantes a partir del primer mes de edad, aunque se halló influido en su duración e intensidad por características individuales o ambientales. Además, estas consistencias en las respuestas se hallaron en distintos países, aunque solo se encontraron dos investigaciones realizadas en Latinoamérica, en Argentina y Ecuador (Gago Galvagno et al., 2019; Handal et al., 2017), y dos en grupos étnicos pertenecientes a China y África (Kisilevsky et al., 1998; Segal et al., 1995).

Las variables de temperamento, NSE y presencia de trastornos mentales en las madres se han asociado a las respuestas típicas en el PSF, si bien aún precisan ser más investigadas, ya que algunos resultados son inconsistentes (Vieites \& Reeb-Sutherland, 2017; Yoo \& Reeb-Sutherland, 2013). Respecto a la edad de aparición de las respuestas típicas al PSF, aparecen claramente a partir del primer mes de vida en todos los bebés evaluados. En resumen, el PSF es un método que provoca un patrón de respuestas robusto en los infantes y niños. 


\section{Conclusiones}

Se concluye que el PSF es un método robusto, ya que (a) provoca respuestas comportamentales y fisiológicas aversivas, relacionadas con el estrés, en todos los niños con desarrollo típico y con expresiones similares según la edad; (b) aparecen a edades tempranas y (c) están reguladas en su intensidad y duración por factores ambientales y por diferencias individuales, sin cambiar su patrón de respuestas.

Con respecto a los factores moduladores, la SM fue sobre la que más estudios y con menos contradicciones entre estos se halló, lo cual podría deberse al fuerte grado de dependencia que posee la regulación emocional infantil en relación a los cuidadores primarios (Bigelow \& Power, 2012). En cuanto a los trastornos de la madre, en la mayoría de los casos afectan la SM, tornándose esta en la variable mediadora entre el PSF y el trastorno (Graham et al., 2018). Con respecto a los resultados del PSF en función del NSE y educativo de los cuidadores primarios, ellos no fueron consistentes, aunque son escasos los trabajos que indagan sobre este tema (Gago Galvagno et al., 2019, Mesman et al., 2009). Acerca de las diferencias individuales, la respuesta al PSF se halló asociada por las dimensiones del temperamento infantil (Gago Galvagno et al., 2019; Yoo \& Reeb-Sutherland; 2013). A pesar de hallarse resultados significativos, aún existen inconsistencias que deben ser solucionadas con más investigaciones en el área. Una forma de medir el temperamento de un modo más válido sería clasificarlo en función de pruebas estandarizadas del comportamiento del infante, ya que las respuestas de los padres o cuidadores no siempre se correlacionaron con observaciones realizadas por especialistas (e.g., Seifer et al., 1994).

Por último, es necesario destacar que una teoría alternativa, no hallada en la literatura previa, aunque no excluyente de las anteriores, es considerar al PSF como un procedimiento adecuado para evaluar el desarrollo evolutivo de la respuesta de los humanos ante el ostracismo, exclusión o frustración social (todas estas denominaciones corresponden a mecanismos similares). La frustración social se define como las respuestas que se desencadenan cuando se disminuye o se omite un reforzador social (e.g., atención, contacto físico o visual, e interacción social) en contextos en los cuales los sujetos tienen la expectativa de recibirlos (Cuenya et al., 2013; Mustaca, 2018). Se halló que las primeras reacciones ante la omisión de reforzadores positivos son incondicionadas, poco reguladas por diferencias individuales y de aprendizaje; en cambio en una segunda etapa están más influenciadas por esos factores (e.g., Amsel, 1992; Karmiola et al., 2019; Williams, 2009), siendo fundamentales a la hora de lograr una buena adaptación durante la vida.

Los resultados de las investigaciones sobre el PSF apoyan esta teoría, ya que este sería un método adecuado para estudiar el desarrollo de los efectos y mecanismos de la exclusión social durante la ontogenia humana. Puede explicar la presencia en todos los niños con desarrollo típico de las respuestas al PSF y la complejidad que implica evaluar qué componentes influyen en su intensidad y duración, ya que existe una compleja interacción entre factores genéticos, epigenéticos y contextuales. Los resultados hallados en este paradigma son, además, congruentes con los experimentos sobre exclusión social evaluados en niños desde los 7 años, con las diferencias propias de la edad evolutiva de cada muestra y de los paradigmas utilizados (e.g., Dvir, 2014; Gago Galvagno et al., 2019; Perry et al., 2011; Wesselmann et al., 2012).

Una ventaja de esta aproximación teórica es que se pueden relacionar resultados hallados en otras etapas del desarrollo en animales sociales con los de los bebés y viceversa, y de adultos, enriqueciendo el campo de ambas líneas de investigación y generando nuevas ideas e hipótesis. Se presentarán dos ejemplos que justifican la relevancia teórica de esta propuesta.

Existe amplia evidencia de que los efectos de la emocionalidad negativa ante la frustración provocada por disminución u omisión de incentivos apetitivos no sociales (e.g., alimento) es una respuesta incondicionada que aparece en los bebés a partir de la cuarta hora de su nacimiento (e.g., Kobre \& Lipsitt, 1972). Esto indica que el desarrollo neural de los humanos está presente desde el nacimiento, como para memorizar el incentivo que reciben en el pasado, generar expectativas de seguir recibiéndolo y expresar con su conducta los cambios repentinos en su calidad o cantidad. Los pocos estudios realizados con el PSF en bebés de menos de un mes mostraron que al cuarto día del nacimiento no se expresa la respuesta (Bertin \& Striano, 2006; Bigelow \& Power, 2012), a los 7 días reaccionan ante el cambio de la contingencia de la fase I a la II, pero no a la del reencuentro en la fase III, y es a partir del primer mes que aparecen las respuestas típicas halladas en las tres fases. La aparición de la respuesta aversiva a los 7 días en la fase II indica que los bebés detectaron el cambio de contingencia y lo pudieron expresar con su conducta. Su continuidad durante la fase III sugiere que o (a) el bebé detecta el cambio de contingencia, pero no es capaz de regular la reacción emocional que le causó la fase II, como lo sugieren los autores (Mesman et al., 2009; Tronick et al., 1978) o (b) no llega a 
detectar su modificación. El modelo teórico de la frustración social apoyaría la primera opción, ya que mostró mecanismos de detección de cambios de incentivos. Esta conjetura se podría poner a prueba, aumentando la duración de la fase III y/o evaluando otras respuestas, por ejemplo, fisiológicas o neurofisiológicas. Además, dado que hay evidencia de que los efectos de frustración por incentivos apetitivos se expresaron al cuarto día de nacimiento, se puede conjeturar que también pueden hallarse respecto de reforzadores sociales y que no se expresa posiblemente debido al escaso desarrollo de otros sistemas, como el motor. Es posible que se necesiten otros métodos o el uso de otras medidas dependientes para detectar la frustración social a edades más tempranas del desarrollo.

Un segundo ejemplo para mostrar el valor de la perspectiva teórica propuesta es el hallazgo de que los bebés de madres depresivas tienen más respuestas de emociones positivas durante las fases II y III respecto de madres no depresivas (Graham et al., 2018). Los autores sugieren que los bebés de madres con depresión pueden habituarse al comportamiento deprimido de sus madres y reaccionar de la manera que consideran apropiada con ella, adoptando un aumento del afecto positivo, ya sea como una estrategia de afrontamiento para reducir la angustia de la experiencia de la fase II o para atraer mejor el apoyo de la madre (Graham et al., 2018). La teoría de la frustración social podría explicarla como una respuesta aprendida mantenida por un programa de reforzamiento intermitente que trae como consecuencia conductas más persistentes ante la violación de expectativas (e.g., Gago Galvagno et al., 2019) o bien por reforzamiento positivo proporcionado por las madres depresivas que, tal vez, respondan con su atención a esas emociones y mucho menos a las neutras o negativas de sus bebés. Todas estas conjeturas pueden someterse a pruebas en el futuro, lo que aumentaría la comprensión del desarrollo de la frustración social, el logro de una adecuada tolerancia a ella y los modos de autorregulación desde edades tempranas.

\section{Limitaciones de las Investigaciones Revisadas}

Las inconsistencias en los resultados de las investigaciones se pueden deber a la falta de control de las variables que podrían estar ejerciendo efectos y que precisan de mayor investigación, como el nivel educativo de la madre, el apoyo social familiar, el tamaño de la familia, los acontecimientos estresantes de la vida, la ocupación del jefe de hogar y la condición de minoría desfavorecida (Sameroff et al., 1993; Smaling et al., 2016).

En cuanto a la metodología, ninguno de los estudios revisados utilizó muestreos de tipo probabilístico. Sin embargo, dado el tipo de investigación, se reconocen las dificultades que entraña realizar muestreos representativos. Además, la mayoría de investigaciones es con infantes de 12 meses y se realizan con las madres de los mismos.

Los estudios longitudinales fueron escasos y las variables moduladoras fueron asignadas, por lo cual las estadísticas utilizadas fueron correlaciones o asociaciones o bien comparaciones entre diferentes grupos. En ese sentido, ninguna de estas variables debe considerarse estrictamente causal.

En cuanto a la presente investigación, la principal limitación fue que solo se evaluaron artículos de revistas, excluyendo las publicaciones en otros tipos de formato (libros, capítulos de libro, congresos u otros). Además, se revisaron artículos publicados en español, inglés y portugués, lo cual introduce un sesgo de idioma.

\section{Direcciones Futuras}

En los casos en que se utilizan test psicométricos para medir las variables sería necesario: (a) que se expliciten los índices de su consistencia interna y su validez; (b) que el temperamento pueda medirse con otras pruebas estandarizadas; (c) que se presente en sus resultados el tamaño del efecto y (d) que los observadores sean independientes a ciegas para evaluar la validez de los resultados.

También son necesarios más estudios con niños de rangos etarios menores, para conocer el momento de aparición de esta respuesta al PSF, y con mayores de 12 meses, para analizar como varían las respuestas al PSF en función de las capacidades que se van adquiriendo con la edad (e.g., lenguaje, motricidad y autorregulación).

Entre otro tipo de investigaciones a realizar, están las relacionadas con variaciones en quién participa en la prueba (madre, padre y extraño) y la duración de cada fase del PSF.

Aunque la realización de diseños experimentales es una limitación que no puede resolverse, estudios multivariados o modelos de ecuaciones estructurales podrían establecer predicciones acerca del porcentaje de la varianza que puede explicar cada variable, los tipos de asociaciones entre ellas y las varianzas de error. 
Sería necesario hacer estudios longitudinales en diferentes países y culturas, para generar un seguimiento, en especial para evaluar cómo va desarrollándose la capacidad de la regulación emocional en niños con desarrollo típico, comparándolos con aquellos que tienen patologías.

Una vez estudiado en mayor profundidad este fenómeno, el PSF sería un método apropiado para detectar problemas en la regulación emocional de los niños y así poder realizar intervenciones tempranas para que aprendan formas más adaptativas de enfrentar eventos estresantes. Teniendo en cuenta la importancia que tiene en la vida aprender a enfrentarse de manera adecuada a situaciones de frustración y de exclusión social, los estudios del PSF pueden arrojar luz sobre las posibles deficiencias en etapas tempranas y evaluar intervenciones para modificarlas. Finalmente, considerar el PSF como un método apropiado para evaluar la frustración social en infantes abre un gran abanico de interrogantes para responder.

\section{Referencias}

Adamson, L. B. \& Frick, J. E. (2003). The still face: A history of a shared experimental paradigm. Infancy, 4(4), 451-473. https://doi.org/10.1207/S15327078IN0404 01

Ainsworth, M. D. S., Blehar, M. C., Waters, E. \& Wall, S. N. (2015). Patterns of attachment: A psychological study of the strange situation. Psychology Press. https://doi.org/10.4324/9780203758045

Aktar, E., Colonnesi, C., de Vente, W., Majdandžić, M. \& Bögels, S. M. (2017). How do parents' depression and anxiety, and infants' negative temperament relate to parent-infant face-to-face interactions? Development and Psychopathology, 29(3), 697-710. https://doi.org/10.1017/S0954579416000390

Amsel, A. (1992). Frustration theory: An analysis of dispositional learning and memory. Cambridge University Press. https://doi.org/10.1017/CBO9780511665561

Apter, G., Devouche, E., Garez, V., Valente, M., Genet, M. -C., Gratier, M., Dominguez, S. \& Tronick, E. (2017). The still-face: A greater challenge for infants of mothers with borderline personality disorder. Journal of Personality Disorders, 31(2), $156-169$. https://doi.org/10.1521/pedi_2016 30_243

Bertin, E. \& Striano, T. (2006). The still-face response in newborn, 1.5-, and 3-month-old infants. Infant Behavior \& Development, 29(2), 294-297. https://doi.org/10.1016/j.infbeh.2005.12.003

Bigelow, A. E. \& Power, M. (2012). The effect of mother-infant skin-to-skin contact on infants' response to the still face task from newborn to three months of age. Infant Behavior and Development, 35(2), 240-251. https://doi.org/10.1016/j.infbeh.2011.12.008

Bigelow, A. E. \& Power, M. (2014). Effects of maternal responsiveness on infant responsiveness and behavior in the still-face task. Infancy, 19(6), 558-584. https://doi.org/10.1111/infa.12059

Braungart-Rieker, J. M., Zentall, S., Lickenbrock, D. M., Ekas, N. V., Oshio, T. \& Planalp, E. (2014). Attachment in the making: Mother and father sensitivity and infants' responses during the still-face paradigm. Journal of Experimental Child Psychology, $125,63-84$. https://doi.org/10.1016/j.jecp.2014.02.007

Busuito, A. \& Moore, G. A. (2017). Dyadic flexibility mediates the relation between parent conflict and infants' vagal reactivity during the face-to-face still-face. Developmental Psychobiology, 59(4), 449-459. https://doi.org/10.1002/dev.21508

Celestino, V. R. R. \& Bucher-Maluschke, J. S. N. F. (2018). Research on systemic psychology in Latin America: An integrative review with methods and data triangulation. Psicologia: Teoria e Pesquisa, 34, Artículo e3443. https://doi.org/10.1590/0102.3772e3443

Conradt, E. \& Ablow, J. (2010). Infant physiological response to the still-face paradigm: Contributions of maternal sensitivity and infants' early regulatory behavior. Infant Behavior \& Development, 33(3), 251-265. https://doi.org/10.1016/j.infbeh.2010.01.001

Coppola, G., Aureli, T., Grazia, A. \& Ponzetti, S. (2016). Reunion patterns in the still-face paradigm as predicted by maternal sensitivity and dyadic coordination. Infancy, 21(4), 453-477. https://doi.org/10.1111/infa.12115

Crandell, L. E., Patrick, M. P. H. \& Hobson, R. P. (2003). 'Still-face' interactions between mothers with borderline personality disorder and their 2-month-old infants. The British Journal of Psychiatry, 183(3), 239-247. https://doi.org/10.1192/bjp.183.3.239

Cuenya, L., Fosacheca, S. E. \& Mustaca, A. (2013). Diferencias individuales en las respuestas de frustración. Revista Argentina de Ciencias del Comportamiento, 5(3), 3-14. https://doi.org/10.32348/1852.4206.v5.n3.5554

DiCorcia, J. A., Snidman, N., Sravish, A. V. \& Tronick, E. (2016). Evaluating the nature of the still-face effect in the double face-to-face still-face paradigm using different comparison groups. Infancy, 21(3), 332-352. https://doi.org/10.1111/infa.12123

Dvir, M. (2014). The effect of ostracism by strangers on romantic relationship evaluations [Tesis de magíster, Purdue University]. Purdue e-Pubs. https://docs.lib.purdue.edu/cgi/viewcontent.cgi?article $=1459 \&$ context=open_access theses

Field, T. M. (1984). Early interactions between infants and their postpartum depressed mothers. Infant Behavior \& Development, 7(4), 517-522. https://doi.org/10.1016/S0163-6383(84)80010-7

Fogel, A. (1982). Affect dynamics in early infancy: Affective tolerance. En T. Field \& A. Fogel (Eds.), Emotion and early interaction (pp. 15-56). Lawrence Erlbaum.

Freund, J. -D. (2019). Early temperament in parental report and scientific observation. Early Child Development and Care, 189(14), 2318-2333. https://doi.org/10.1080/03004430.2018.1450252

Gago Galvagno, L. G., De Grandis, M. C., Clerici, G. D., Mustaca, A. E., Miller, S. E. \& Elgier, A. M. (2019). Regulation during the second year: Executive function and emotion regulation links to joint attention, temperament, and social vulnerability in a Latin American sample. Frontiers in Psychology, 10, Artículo 1473. https://doi.org/10.3389/fpsyg.2019.01473

Giusti, L., Provenzi, L. \& Montirosso, R. (2018). The face-to-face still-face (FFSF) paradigm in clinical settings: Socio-emotional regulation assessment and parental support with infants with neurodevelopmental disabilities. Frontiers in Psychology, 9, Artículo 789. https://doi.org/10.3389/fpsyg.2018.00789

Graham, K. A., Blissett, J., Antoniou, E. E., Zeegers, M. P. \& McCleery, J. P. (2018). Effects of maternal depression in the still-face paradigm: A meta-analysis. Infant Behavior \& Development, 50, 154-164. https://doi.org/10.1016/j.infbeh.2017.12.001

Grant, K. -A., McMahon, C., Austin, M. -P., Reilly, N., Leader, L. \& Ali, S. (2009). Maternal prenatal anxiety, postnatal caregiving and infants' cortisol responses to the still-face procedure. Developmental Psychobiology, 51(8), 625-637. https://doi.org/10.1002/dev.20397 
Grant, K. -A., McMahon, C., Reilly, N. \& Austin, M. -P. (2010). Maternal sensitivity moderates the impact of prenatal anxiety disorder on infant mental development. Early Human Development, 86(9), 551-556. https://doi.org/10.1016/j.earlhumdev.2010.07.004

Gunning, M., Halligan, S. L. \& Murray, L. (2013). Contributions of maternal and infant factors to infant responding to the still face paradigm: A longitudinal study. Infant Behavior and Development, 36(3), 319-328. https://doi.org/10.1016/j.infbeh.2013.02.003

Haltigan, J. D., Leerkes, E. M., Supple, A. J. \& Calkins, S. D. (2014). Infant negative affect and maternal interactive behavior during the still-face procedure: The moderating role of adult attachment states of mind. Attachment \& Human Development, 16(2), 149-173. https://doi.org/10.1080/14616734.2013.863734

Handal, A. J., Garcia Saavedra, L., Schrader, R., Aragón, C. L., Páez, M. \& Lowe, J. R. (2017). Assessment of maternal-infant interaction: Application of the still face paradigm in a rural population of working women in Ecuador. Maternal and Child Health Journal, 21(3), 458-466. https://doi.org/10.1007/s10995-016-2123-9

Kaitz, M., Maytal, H. R., Devor, N., Bergman, L. \& Mankuta, D. (2010). Maternal anxiety, mother-infant interactions, and infants' response to challenge. Infant Behavior \&Development, 33(2), 136-148. https://doi.org/10.1016/j.infbeh.2009.12.003

Karmiola, S., Cuenya, L. \& Mustaca, A. E. (2019). Comprendo y siento tu dolor: efectos emocionales de viñetas sobre exclusión social en adultos. Acta de Investigación Psicológica, 9(1), 108-118. https://doi.org/10.22201/fpsi.20074719e.2019.1.10

Kisilevsky, B. S., Hains, S. M. J., Lee, K., Muir, D. W., Xu, F., Fu, G., Zhao, Z. Y. \& Yang, R. L. (1998). The still-face effect in Chinese and Canadian 3- to 6-month-old infants. Developmental Psychology, 34(4), 629-639. https://doi.org/10.1037/0012-1649.34.4.629

Kobre, K. R. \& Lipsitt, L. P. (1972). A negative contrast effect in newborns. Journal of Experimental Child Psychology, 14(1), 81-91. https://doi.org/10.1016/0022-0965(72)90033-1

Kogan, N. \& Carter, A. S. (1996). Mother-infant reengagement following the still-face: The role of maternal emotional availability an infant affect regulation. Infant Behavior \& Development, 19(3), 359-370. https://doi.org/10.1016/S0163-6383(96)90034-X

Lowe, J. R., Coulombe, P., Moss, N. C., Rieger, R. E., Aragón, C., MacLean, P. C., Caprihan, A., Phillips, J. P. \& Handal, A. J. (2016). Maternal touch and infant affect in the still face paradigm: A cross-cultural examination. Infant Behavior \& Development, 44, 110-120. https://doi.org/10.1016/j.infbeh.2016.06.009

Lowe, J. R., MacLean, P. C., Duncan, A. F., Aragón, C., Schrader, R. M., Caprihan, A. \& Phillips, J. P. (2012). Association of maternal interaction with emotional regulation in 4- and 9-month infants during the still face paradigm. Infant Behavior \& Development, 35(2), 295-302. https://doi.org/10.1016/j.infbeh.2011.12.002

MacLean, P. C., Rynes, K. N., Aragón, C., Caprihan, A., Phillips, J. P. \& Lowe, J. R. (2014). Mother-infant mutual eye gaze supports emotion regulation in infancy during the still-face paradigm. Infant Behavior \& Development, 37(4), 512-522. https://doi.org/10.1016/j.infbeh.2014.06.008

Mantis, I., Mercuri, M., Stack, D. M. \& Field, T. M. (2019). Depressed and non-depressed mothers' touching during social interactions with their infants. Developmental Cognitive Neuroscience, 35, 57-65. https://doi.org/10.1016/j.dcn.2018.01.005

Mastergeorge, A. M., Paschall, K., Loeb, S. R. \& Dixon, A. (2014). The still-face paradigm and bidirectionality: Associations with maternal sensitivity, self-esteem and infant emotional reactivity. Infant Behavior and Development, 37(3), 387-397. https://doi.org/10.1016/j.infbeh.2014.05.006

Mesman, J., van IJzendoorn, M. H. \& Bakermans-Kranenburg, M. J. (2009). The many faces of the still-face paradigm: A review and meta-analysis. Developmental Review, 29(2), 120-162. https://doi.org/10.1016/j.dr.2009.02.001

Miller, S. E. \& Marcovitch, S. (2015). Examining executive function in the second year of life: Coherence, stability, and relations to joint attention and language. Developmental Psychology, 51(1), 101-114. https://doi.org/10.1037/a0038359

Moore, G. A., Hill-Soderlund, A. L., Propper, C. B., Calkins, S. D., Mills-Koonce, W. R. \& Cox, M. J. (2009). Mother-infant vagal regulation in the face-to-face still-face paradigm is moderated by maternal sensitivity. Child Development, 80(1), 209-223. https://doi.org/10.1111/j.1467-8624.2008.01255.x

Müller, M., Tronick, E., Zietlow, A. -L., Nonnenmacher, N., Verschoor, S. \& Träuble, B. (2016). Effects of maternal anxiety disorders on infant self-comforting behaviors: The role of maternal bonding, infant gender and age. Psychopathology, 49(4), 295-304. https://doi.org/10.1159/000448404

Musser, E. D., Ablow, J. C. \& Measelle, J. R. (2012). Predicting maternal sensitivity: The roles of postnatal depressive symptoms and parasympathetic dysregulation. Infant Mental Health Journal, 33(4), 350-359. https://doi.org/10.1002/imhj.21310

Mustaca, A. E. (2018). Frustración y conductas sociales. Avances en Psicología Latinoamericana, 36(1), 65-81. https://doi.org/10.12804/revistas.urosario.edu.co/apl/a.4643

Noe, D., Schluckwerder, S. \& Reck, C. (2015). Influence of dyadic matching of affect on infant self-regulation. Psychopathology, 48(3), 173-183. https://doi.org/10.1159/000376586

Nunnaly, J. C. (1978). Psychometric theory (2a ed.). McGraw-Hill.

Oppenheimer, J. E., Measelle, J. R., Laurent, H. K. \& Ablow, J. C. (2013). Mothers' vagal regulation during the still-face paradigm: Normative reactivity and impact of depression symptoms. Infant Behavior \& Development, 36(2), 255-267. https://doi.org/10.1016/j.infbeh.2013.01.003

Perry, Y., Henry, J. D., Sethi, N. \& Grisham, J. R. (2011). The pain persists: How social exclusion affects individuals with schizophrenia British Journal of Clinical Psychology, 50(4), 339-349. https://doi.org/10.1348/014466510X523490

Planalp, E. M., Braungart-Rieker, J. M., Lickenbrock, D. M. \& Zentall, S. R. (2013). Trajectories of parenting during infancy: The role of infant temperament and marital adjustment for mothers and fathers. Infancy, 18(s1), E16-E45. https://doi.org/10.1111/infa.12021

Ramsay, D. \& Lewis, M. (2003). Reactivity and regulation in cortisol and behavioral responses to stress. Child Development, 74(2), 456-464. https://doi.org/10.1111/1467-8624.7402009

Reck, C., Tietz, A., Müller, M., Seibold, K. \& Tronick, E. (2018). The impact of maternal anxiety disorder on mother-infant interaction in the postpartum period. PLoS ONE, 13(5), Artículo e0194763. https://doi.org/10.1371/journal.pone.0194763

Sameroff, A. J., Seifer, R., Baldwin, A. \& Baldwin, C. (1993). Stability of intelligence from preschool to adolescence: The influence of social and family risk factors. Child Development, 64(1), 80-97. https://doi.org/10.1111/j.1467-8624.1993.tb02896.x

Segal, L. B., Oster, H., Cohen, M., Caspi, B., Myers, M. \& Brown, D. (1995). Smiling and fussing in seven-month-old preterm and fullterm black infants in the still-face situation. Child Development, 66(6), 1829-1843. https://doi.org/10.1111/j.1467-8624.1995.tb00968.x

Seifer, R., Sameroff, A. J., Barrett, L. C. \& Krafchuk, E. (1994). Infant temperament measured by multiple observations and mother report. Child Development, 65(5), 1478-1490. https://doi.org/10.1111/j.1467-8624.1994.tb00830.x 
Seixas, Í., Barbosa, M. \& Fuertes, M. (2017). Contributos para a auto-regulação do bebé no paradigma face-to-face still-face [Contribuciones a la autorregulación del bebé en el paradigma face-to-face still-face]. Análise Psicológica, 35(4), 469-485. https://doi.org/10.14417/ap.1280

Smaling, H. J. A., Huijbregts, S. C. J., Suurland, J., van der Heijden, K. B., Mesman, J., van Goozen, S. H. M. \& Swaab, H. (2016). Prenatal reflective functioning and accumulated risk as predictors of maternal interactive behavior during free play, the still-face paradigm, and two teaching tasks. Infancy, 21(6), 766-784. https://doi.org/10.1111/infa.12137

Spinelli, M. \& Mesman, J. (2018). The regulation of infant negative emotions: The role of maternal sensitivity and infant-directed speech prosody. Infancy, 23(4), 502-518. https://doi.org/10.1111/infa.12237

Toda, S. \& Fogel, A. (1993). Infant response to the still-face situation at 3 and 6 months. Developmental Psychology, 29(3), 532-538. https://doi.org/10.1037/0012-1649.29.3.532

Trevarthen, C. (1977). Descriptive analyses of infant communicative behavior. En H. R. Schaffer (Ed.), Studies in mother-infant interaction (pp. 227-270). Academic Press.

Tronick, E., Als, H., Adamson, L., Wise, S. \& Brazelton, T. B. (1978). The infant's response to entrapment between contradictory messages in face-to-face interaction. Journal of the American Academy of Child Psychiatry, 17(1), 1-13. https://doi.org/10.1016/S0002-7138(09)62273-1

Vieites, V. \& Reeb-Sutherland, B. C. (2017). Individual differences in non-clinical maternal depression impact infant affect and behavior during the still-face paradigm across the first year. Infant Behavior \& Development, 47, 13-21. https://doi.org/10.1016/j.infbeh.2017.02.005

Wagner, N. J., Mills-Koonce, W. R., Propper, C. B., Willoughby, M. T., Rehder, P. D., Moore, G. A. \& Cox, M. J. (2016). Associations between infant behaviors during the face-to-face still-face paradigm and oppositional defiant and callous-unemotional behaviors in early childhood. Journal of Abnormal Child Psychology, 44(8), 1439-1453. https://doi.org/10.1007/s10802-016-0141-0

Weinberg, M. K., Beeghly, M., Olson, K. L. \& Tronick, E. (2008). A still-face paradigm for young children: $2^{1 / 2}$ year-olds' reactions to maternal unavailability during the still-face. Journal of Developmental Processes, 3(1), 4-21. https:/www.ncbi.nlm.nih.gov/pmc/articles/PMC3289403/

Werner, K. \& Gross, J. J. (2010). Emotion regulation and psychopathology: A conceptual framework. En A. M. Kring \& D. M. Sloan (Eds.), Emotion regulation and psychopathology: A transdiagnostic approach to etiology and treatment (pp. 13-37). Guilford Press.

Wesselmann, E. D., Wirth, J. H., Mroczek, D. K. \& Williams, K. D. (2012). Dial a feeling: Detecting moderation of affect decline during ostracism. Personality and Individual Differences, 53(5), 580-586. https://doi.org/10.1016/j.paid.2012.04.039

Williams, K. D. (2009). Ostracism: A temporal need-threat model. Advances in Experimental Social Psychology, 41, $275-314$. https://doi.org/10.1016/S0065-2601(08)00406-1

Yoo, K. \& Reeb-Sutherland, B. C. (2013). Effects of negative temperament on 5-month-old infants' behavior during the still-face paradigm. Infant Behavior \& Development, 36(3), 344-348. https://doi.org/10.1016/j.infbeh.2013.03.002

Fecha de recepción: Noviembre de 2019.

Fecha de aceptación: Julio de 2020. 